\title{
Intervención en estrategias de comprensión: un modelo basado en el conocimiento y aplicación de la estructura del texto
}

\author{
JOSÉ A. LEÓN \\ Universidad Autónoma de Madrid

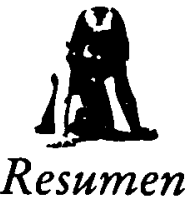

En dos estudios complementarios se analizó la implantación y posterior evaluación de un programa de instrucción en alumnos con diferente grado de babilidad lectora. Este programa incidia básicamente en estrategias que requerian para su aplicación un conocimiento expreso sobre la estructura del texto. En un primer estudio, los 72 alumnos de $1 .^{\circ}$ de BUP que tomaron parte (36 con buen nivel y 36 con un pobre nivel de comprensión) se distribuyeron equitativamente en tres grupos: un grupo experimental y dos grupos control. A todos los grupos se aplicaron dos pruebas de recuerdo libre: inmediato y demorado ( 7 dias). Los resultados indicaron una mejora sustantiva de los lectores que fueron entrenados en este programa respecto a sus compañeros homólogos de los grupos control, en la comprensión y recuerdo de la información relevante (macroestructura). En un segundo estudio, se evaluó el grado de transferencia de lo aprendido a situaciones nuevas. Los resultados indicaron que los sujetos entrenados en este programa experimental mostraron esta misma babilidad para organizar su recuerdo en textos cuya estructura organizativa no babia sido previamente instruida.

Palabras clave: Comprensión de textos; Comprensión lectora; Programas de instrucción; Estrategias de aprendizaje; Textos expositivos.

\section{Intervention in comprehension strategies: a model based con knowledge and use of the text structure}

\section{Abstract}

In two complimentary studies, the implantation and later the evaluation of an instructional program of high school students with different reading skills was analyzed. This program basically influenced the strategies that were required for its application of an explicit knowledge about the structure of the text. In the first study, the 72 junior bigh school students that participated (36 with a good level and 36 subjects with a poor reading comprenbension level) were evenly distributed into three groups: experimental and two control groups. In order to evaluate the performance of the groups, two tests of free recall, immediate and delayed (seven days), were given. The results indicated a substantial improvement of the readers who were trained in this program in respect to their homologous partnes in the control group in the comprehension and recall of the relevant information. In the second study, the trained subjects in experimental program transfered their new knowledge to text whose organizational structure hat not been previously instructed.

Key words: Text comprehension; Reading comprehension; Instruction program; Learning strategies; Comprehension strategies; Expository text.

Agradecimientos: Deseamos expresar nuestro agradecimiento a los alumnos y a la dirección del I.N.B. «Rey Pastor» por su colaboración y participación en este estudio.

Dirección del autor: Universidad Autónoma de Madrid. Facultad de Psicología. Departamento de Psicología Básica Social y Metodología. Campus de Cantoblanco, 28049 Madrid. 1991.

Original recibido: Noviembre 1990. Revisión recibida: Junio 1991. Aceptado: Septiembre 


\section{INTRODUCCION}

Investigaciones recientes sobre el tema que nos ocupa nos sugieren que los buenos lectores se muestran más activos que los lectores con pobre nivel de comprensión cuando procesan la información del texto. Estas diferencias se producen frecuentemente, porque, al contrario que los lectores más consumados, los menos competentes no son capaces de organizar correctamente la información nueva, lo que les imposibilita relacionarla con la que ya se posee. En muchos casos estas diferencias pueden estar motivadas por la ineficacia o inhabilidad que muestran estos lectores a la hora de seleccionar e implementar las estrategias adecuadas en la comprensión del material escrito.

Desde distintas ópticas, diversos autores han especificado algunas estrategias en un intento de caracterizar a los lectores competentes y menos competentes. En general, todos coinciden en alguna medida con las características globales de la representación que obtienen los diferentes sujetos en su memoria tras la lectura de un texto expositivo. Frente a la representación mental de los lectores consumados, caracterizada por una "representación coherente» que distingue diferentes niveles de importancia en el contenido del texto, reteniendo las ideas que determinan la macroestructura del mismo, la de los lectores menos competentes, por el contrario, se presenta bajo la forma «tema más detalles" (Scardamalia y Bereiter, 1984). Esta última se caracteriza por una representación mental del tema general del texto acompañada por una colección inarticulada de detalles.

Así, desde esta perspectiva, se han identificado estrategias propias de los lectores competentes y que han sido denominadas de diferentes formas, estrategias "organizativas" (Just y Carpenter, 1987), de "comprensión" (Spring, 1985), "estrategia significativa" (meaninful strategy) (Loman y Mayer, 1983), "codificación asimilativa» (Mayer, 1985), o "estrategia estructural" (Meyer, 1984). Estas estrategias están encaminadas a generar o activar los esquemas que posee el sujeto, requieren en el lector la existencia de un conocimiento previo adecuado y de la disponibilidad de un uso estratégico de esos conocimientos.

Dentro de estas estrategias podría incluirse aquella destreza que permite al lector relacionar la nueva información leída con la que ya posee, permitiéndole inferir información no explicitada en el texto o aquella habilidad para identificar las principales relaciones lógicas del pasaje, que permitan conectar las diferentes proposiciones del texto dentro de una secuen- cia coherente. Esta noción sobre la estructura del texto, permite al lector consumado la construcción de una representación o modelo mental de la información leída más coherente y organizada (Mayer, 1985). De esta forma, el lector comprende el pasaje sin necesidad de recordar la información específica. La estrategia estructural, por ejemplo, requiere que el lector experto posea un conocimiento sobre cómo están organizados convencionalmente los textos y una estrategia que le permite buscar y utilizar la estructura de alto nivel en un texto particular, que actúa como marco organizacional donde se codifica y recupera la información (Meyer, 1984).

Por el contrario, el lector inmaduro presenta otros tipos de estrategias, como la "estrategia mecánica» (Loman y Mayer, 1983), «estrategia en lista por defecto" (Meyer, 1984) o ante tareas de resumen, la estrategia «suprimir y copiar" propuesta por Brown, Day y Jones (1983). Estas estrategias se caracterizan por una representación fragmentada y lineal y se utilizan cuando el lector concibe el pasaje como una lista de hechos o acontecimientos separados que han de ser memorizados. La estrategia en lista por defecto, por ejemplo, describe la situación de un lector que, aun pretendiendo extraer la misma representación que la propuesta por el autor, carece de un plan estratégico de procesamiento. En este caso, el lector parece operar como si la meta de la lectura fuese recordar «algo» del texto, lo que podría comprobarse en el protocolo de recuerdo como una relativa insensibilidad a la organización jerárquica del contenido textual. En consecuencia, el lector menos competente concibe el texto como una lista de elementos aislados, careciendo de toda organización lógica (Meyer, 1984).

La presencia de estos tipos de estrategias ha sido puesta de manifiesto en trabajos recientes dirigidos a analizar diferencias entre lectores «expertos» y «novicios» (León, 1989; León y Carretero, 1990). Los resultados obtenidos en estos estudios indican que las diferencias esenciales entre las respuestas emitidas por los adultos expertos y alumnos de $10^{\circ}$ de BUP aparecen en la calidad de las respuestas, siendo las del experto más selectivas respecto a la macroestructura del texto y mejor organizadas desde el punto de vista de la estructura original del pasaje. Este conocimiento implícito sobre la estructura del texto se revela como uno de los factores claves responsables de esta superioridad. El lector maduro parece utilizar hábilmente este conocimiento tanto en la representación de la nueva información como en la recuperación de esa información a la hora de elaborar sus respuestas. Al mismo tiempo, el 
uso de este tipo de conocimiento revierte en una mayor estabilidad del recuerdo con el paso del tiempo.

Estas diferencias nos sugieren a la hora de planificar un modelo de instrucción que incida en la mejora de la comprensión del material escrito en lectores menos competentes, basarnos en la asunción de que estos lectores pueden beneficiarse de la enseñanza de aquellas estrategias que los lectores maduros utilizan de forma natural (Oakhil y Garnham, 1988). Más específicamente, si se interviene en procedimientos específicos que induzcan al lector un conocimiento sobre la estructura textual y su utilización, así como la implantación de sistemas de control de las propias actividades que pretenden promoverse, pueden mejorar el comportamiento lector en aquellos sujetos que no poseen estrategias de comprensión o si las poseen, no las utilizan adecuadamente. En este sentido, podemos presumir que el comportamiento del lector menos capaz se aproximaría en tal caso al realizado por el lector consumado.

\section{ESTUDIOS INSTRUCCIONALES}

\section{Estudio 1}

\section{PLANTEAMIENTO DE LA INVESTIGACION}

El propósito principal de esta investigación fue comprobar si este conocimiento sobre la estructura del texto, especialmente el relativo a un conocimiento expreso de las relaciones lógicas que gobiernan la información textual, así como las estrategias que inciden en la detección de la macroestructura, presentes en los adultos expertos (véase León b, en esta misma revista) podrían ser enseñadas mediante una instrucción directa, explícita y sistemática en los lectores más jóvenes. Se pretendia comprobar, asimismo, si ello revertiría en una mejora del producto lector tanto en los adolescentes competentes como en los menos capaces. Sobre este planteamiento diseñamos un programa de intervención dirigido a lectores adolescentes $\left(1 .^{\circ} \mathrm{BUP}\right)$ y que denominamos Programa de Intervención Directa (ID).

Como aspectos complementarios a este propósito principal, deseábamos confirmar experimentalmente si la introducción de señalizaciones en el texto supone una ayuda a la intervención. Al igual que en otros estudios previos, optamos por introducir dos tareas de recuerdo, recuerdo libre inmediato y demorado. A continuación se describe dicho programa.

\section{PROGRAMA DE INTERVENCION DIRECTA (ID)}

\section{A) Objetivos del programa instruccional}

Con este entrenamiento se pretendía mejorar la comprensión final del material escrito en los alumnos de BUP a través de la enseñanza de algunas estrategias que les permitieran reconocer la estructura de un texto, desvelar sus relaciones y operar esa estructura para reconstruir el texto en la memoria. Más específicamente, se trataba de adiestrarles en habilidades que les permitiesen:

- Obtener un conocimiento específico sobre la estructura de algunos textos expositivos.

- Activar o generar el esquema general adecuado donde ubicar la información leída.

- Extraer las ideas principales del texto (la macroestructura).

- Identificar y retener las relaciones lógicas esenciales del contenido del pasaje y sus elementos principales.

- Ser capaz de elaborar un resumen adecuado, siguiendo la estructura original del texto.

El programa toma y refunde diversas concepciones o modelos de instrucción (véase tabla I). Por un lado, tomamos el «plan estratégico" propuesto por Meyer y cols. (Bartlett, 1978; Meyer, 1984; 1985a; Meyer y cols., 1989) como principal estrategia a seguir, introducida explícitamente en la segunda tarea del programa. Por otro, lo aplicamos dentro de un contexto social de interacción y bajo la instrucción razonada de habilidades propuesta por Brown y Palincsar (1982; Palincsar y Brown, 1984). Como complemento a estas dos perspectivas, este programa incluye, además, algunas tareas, como la titulación y el resumen, que han sido ampliamente utilizadas y difundidas, tanto en la instrucción de habilidades' aisladas como en programas generales y completos.

B) Descripción de las tareas de este programa de instrucción directa en habilidades lectoras

Para llevar a cabo los objetivos que se pretenden alcanzar, el programa de instrucción incluye las siguientes tareas o actividades.

\section{B.1. Elaboración del título del pasaje}

Ninguno de los textos que se utilizaron como material de trabajo para este programa tenía título. La tarea del lector consistía en elaborar el título que mejor se adecuase a la in- 
TABLA I

Caracteristicas del modelo de intervención directa (ID)

1) QUE INSTRUIR: OBJETIVOS Y TAREAS DEL PROGRAMA

\begin{tabular}{|c|c|c|}
\hline Objetivos & Tareas & Fuentes \\
\hline - Activar esquema general & $-\underset{\text { (Preguntas) }}{\text { Titulación }}$ & Williams y cols., 1981. \\
\hline $\begin{array}{l}\text { - Conocer la organización } \\
\text { interna de algunos textos } \\
\text { expositivos } \\
\text { (Elaboración de un Esque- } \\
\text { ma estructural) }\end{array}$ & & $\begin{array}{l}\text { Meyer, 1984, 1985a; Bart- } \\
\text { lett, 1978; Brooks y Dan- } \\
\text { sereau, 1983. }\end{array}$ \\
\hline \multirow[t]{3}{*}{$\begin{array}{l}\text { - Aplicar *Plan estratégico» } \\
\text { Activar «Esquema estruc- } \\
\text { tural» }\end{array}$} & $\begin{array}{l}\text { - A) Identificar la relación } \\
\text { retórica dominante y sus } \\
\text { elementos principales } \\
\text { (Preguntas) }\end{array}$ & $\begin{array}{l}\text { Bartlett, 1978; Meyer, } \\
\text { 1984; Meyer y cols., } 1989 .\end{array}$ \\
\hline & $\begin{array}{l}\text { - B) Extraer las ideas rele- } \\
\text { vantes contenidas en ellas. } \\
\text { (Recuadros de gráficos) }\end{array}$ & Geva, 1983; León, 1989. \\
\hline & $\begin{array}{l}\text { - C) Elaborar un resumen } \\
\text { escrito siguiendo la estruc- } \\
\text { tura interna principal del } \\
\text { texto. } \\
\text { (Resumen) }\end{array}$ & $\begin{array}{l}\text { Brown y col., 1983; Tay- } \\
\text { lor, 1980; Taylor y Beach, } \\
\text { 1984. }\end{array}$ \\
\hline
\end{tabular}

2) COMO INSTRUIR: PROCEDIMIENTO DE LA INTERVENCION DIRECTA

- APLICACION DE LA INSTRUCCION RAZONADA DE HABILIDADES (Brown y Palincsar, 1982; Palincsar y Brown, 1984)

FASES: A) EXPLICACION: Descripción razonada de cada una de las tareas, utilidad y finalidad de las mismas.

B) MODELADO: Representación de la tarea por el profesor con la intención de descargar progresivamente la responsabilidad en el alumno.

C) PRACTICA SUPERVISADA: Regulación de la aplicación de las tareas.

formación que se acababa de leer. Ello le exigía en primer lugar, iniciar la búsqueda de un esquema general que, activado de abajo-arriba (bottom-up), tratase de recoger las características más importantes de la información leída.

Para introducir al sujeto en la tarea, se le sugería que respondiese, una vez leido el texto, a la pregunta, «¿Sobre qué trata el texto?». En el caso que se considerara necesario, se recurría a un material de apoyo que consistia en la presentación de cuatro posibles títulos que una vez escritos en la pizarra, se les pedía a los alumnos que optasen por el que considerasen el correcto. Cada uno de los posibles títulos se refería a una característica del contenido del pasaje. Siguiendo el trabajo de Williams y cols. (1981), las cuatro alternativas que se elaboraron respondían a una de las siguientes características: Una al tema general y otra al tema es- pecífico del pasaje; en una tercera el título era sugerido por la información complementaria y por último, se optaba por un título sobre un tema anómalo que nada tenía que ver con el contenido del texto. El sentido de incluir esta tarea no era otra que la de enseñar a discriminar a los sujetos el tema específico del resto de los títulos sugeridos.

\section{B.2. Identificar la relación dominante del contenido del pasaje y sus elementos principales}

Con esta tarea se introduce al alumno en el conocimiento y uso de la organización interna del texto, a través de algunas de las relaciones retóricas dominantes descritas por $\mathrm{Me}$ yer, $(1984,1985 a)$, características del material expositivo. Estas fueron: Respuesta: proble- 


\section{1}

$\mathrm{ma} /$ solución, Causación: antecedente/consecuente, Colección y Descripción.

Bajo la relación retórica de Respuesta, las ideas del texto frecuentemente están organizadas en dos partes igualmente importantes: El problema y la solución. Esta última como parte que responde al problema como un intento de resolverlo. También puede aparecer bajo la forma de pregunta y respuesta. En el caso de la Causación, las principales ideas están ligadas por una relación causal, donde unas pertenecen a la causa o antecedente y otra a su efecto o consecuente. Las ideas que responden a la Descripción suelen ser ideas que ofrecen una mayor información sobre el tema que trata el texto. Son los casos que se refieren a los atributos, detalles, ejemplos o a la información introductoria. Por último, en la Colección, las ideas se agrupan en torno a una relación común. Una de las más frecuentes es la secuencia, bajo la cual las ideas se agrupan en base a un criterio de orden o de tiempo. La idea principal es el procedimiento o la historia relatada.

Todas estas relaciones retóricas fueron explicadas individualmente con ayuda de ejemplos sencillos. Como forma de orientar a los lectores en la detección de estas relaciones entre ideas y discriminarla del contenido menos relevante, se les sugirió que respondiesen a preguntas que utilizaron como material de apoyo, tales como «¿Cuál de las relaciones estudiadas puede ser tomada en este texto como la principal?, ¿Existe alguna relación principal? ¿Cuáles son sus elementos principales? ¿Hay alguna información de detalle? ¿Se trata ésta de un ejemplo?...

Una vez identificada la relación dominante y sus principales elementos, el lector debía extraer dicha relación. Para ello, el alumno se ayudaba de unos recuadros gráficos que, colocados en orden jerárquico según la estructura de cada texto de trabajo, debía situarla en su lugar correspondiente y completar, asimismo, la tarea, añadiendo en los diferentes recuadros los elementos principales y la información correspondiente a cada uno de ellos (véase anexo).

\section{B.3. Elaborar un resumen del contenido leido}

Con este último requisito se pretendía, además de asentar lo descrito hasta ahora, enseñar al adolescente a aplicar lo aprendido en la elaboración de un resumen. La tarea consistió en elaborar un extracto escrito del contenido del pasaje, siguiendo lo anotado en el esquema gráfico realizado. El lector, por tanto, utilizando tan sólo el título y la información esquemática en la que no debía considerar la información de detalle, debería realizar una síntesis por escrito que tratara de recoger exclusivamente lo más importante del texto. De esta manera, nos asegurábamos que el lector seguía el esquema organizativo del texto en la elaboración de sus respuestas.

\section{C) Cómo instruir: Procedimiento del programa de intervención directa}

Para la implantación de este programa en cada tarea se siguieron los pasos de la «Instrucción Razonada» de cada habilidad descrita por Brown y cols. (Brown y Palincsar, 1982; Palincsar y Brown, 1984) y constaba de tres fases consecutivas: fase de explicación, modelado y práctica supervisada, que describimos a continuación.

1. Fase de explicación. El objetivo de esta fase no era otro que el de describir a los sujetos cada una de las tareas, su utilidad y finalidad de las mismas.

2. Modelado. En esta fase se pretendía modular cada actividad en los alumnos. Primero, a través de la ejecución del profesor para que, de manera progresiva, el alumno fuera internalizando la ejecución asumiendo mayor responsabilidad en la tarea.

3. Práctica supervisada. En esta fase, se trataba de regular la aplicación en cada una de las tareas. El papel del investigador consistió en orientar al alumno en la realización de la tarea, señalando las cuestiones o errores que éste cometía, sugiriendo posibles estrategias, induciéndole a reflexionar y justificar sus decisiones, así como reforzar sus logros. A medida que estos lectores adolescentes conseguían dominar la tarea, se les retiraba paulatinamente las ayudas prestadas por el experimentador.

En la instrucción de cada actividad el experimentador se apoyaba, además, de preguntas previas, cuadros gráficos y ejemplos sencillos para facilitar su aprendizaje.

\section{METODO}

\subsection{Sujetos}

Los sujetos que participaron en este estudio fueron seleccionados de entre 209 alumnos que cursaban en ese momento $10^{\circ}$ de BUP en el Instituto Público de Bachillerato "Rey Pastor», de Madrid. La elección de este nivel para la presente investigación se hizo atendiendo a varias razones. Una de ellas fue la de considerar que, como muestran numerosos trabajos sobre el pensamiento formal, es en estas edades donde se alcanza la plena capacidad para comprender y asimilar conosimientos complejos expresados lingüísticamente y ello nos permite trabajar con materiales relativamente complejos como los 


\section{2}

utilizados en la investigación que aquí se presenta (Carretero y León, 1990). Otra razón no menos importante, hace referencia a que en este período, la capacidad de comunicación significativa está suficientemente desarrollada, a la vez que se han automatizado la estrategias necesarias para la codificación de la información y se van asentando las estrategias semánticas que van a incidir directamente en el significado del mensaje. Todo ello permite analizar los procesos y estrategias que los distintos grupos de sujetos seleccionados presumiblemente utilizan, contrastando así, las posibles diferencias existentes entre ellos (León y García Madruga, 1989).

Puesto que nos interesaba seleccionar a estos alumnos con un claro nivel de competencia lectora, bueno y pobre, todos los sujetos se sometieron a varios criterios: a) una prueba de comprensión lectora (Lázaro, 1982) utilizada recientemente como prueba de comprensión en la investigación de C.I.D.E. para evaluar la reforma de las E.E.M.M. y cuyo baremo de 1 . $^{\circ}$ de BUP utilizamos en el cómputo de los resultados; b) una valoración sobre el rendimiento académico del alumno por los profesores de las asignaturas de Matemáticas, Lengua, Naturaleza e Historia. Con este cuestionario se juzgaba la capacidad y rendimiento académico de sus alumnos en una escala de cinco puntos (muy bueno, bueno, normal, bajo y muy bajo); y c), una tarea resumen que, ante la lectura previa de un texto corto, todos los sujetos debían resumirlo en un máximo de 10 renglones. A través de esta tarea se evaluaba la presencia o ausencia de las ideas importantes del texto y su coherencia global.

La muestra final se obtuvo cruzando estas tres puntuaciones, con el fin de garantizar la comparación entre sujetos con dos niveles de competencia. A esta selección hay que descontar, además, aquellos sujetos en lo que se presumía un bajo nivel intelectual o problemas generales de adaptación. Se tuvo en cuenta, asimismo, sólo a aquellos alumnos que cursaban por vez primera ese nivel académico, obviando en la muestra a los repetidores, por considerar que podían estar familiarizados con los contenidos, podían mostrar conductas poco participativas o podrian establecer importantes diferencias de edad. La asistencia a las sesiones de los distintos tratamientos se consideró como otro de los criterios a tener en cuenta a la hora de computar los datos. Se exigía que el alumno asistiese al menos al $80 \%$ de las sesiones impartidas.

Con todo, se seleccionaron finalmente 72 alumnos que diferian en su nivel de competencia lectora, 36 con un buen nivel de comprensión lectora (BC) y 36 con pobre nivel de comprensión (MC).

\subsection{Tratamientos}

Los sujetos de cada nivel de competencia se asignaron de manera aleatoria a uno de los tres grupos que recibieron una de estas tres condiciones. Un primer grupo a la condición de «intervención directa" (ID), ya descrita. Un segundo, asumió la condición de «intervención control»(IC). Este grupo, a diferencia del anterior, no se instruyó a los sujetos en ninguna estrategia o tarea encaminada a una mejora en el conocimiento en la estructura del texto o en una descripción razonada de habilidades. Muy al contrario, en esta intervención se reforzaba indiscriminadamente la estrategia utilizada por cada alumno, fuese cual fuese ésta. Por último, un tercer grupo asumió la condición de «sin intervención control» (SIC). Este grupo sólo recibió la aplicación de las V.V.D.D.

\subsection{Procedimiento y materiales aplicados en la Intervención ID e IC}

Todo el período de instrucción se llevó a cabo en tres semanas durante el segundo trimestre académico y en siete sesiones (las dos últimas se dedicaron a la evaluación). La intervención se realizó en el aula con todos los alumnos de la clase, que oscilaban entre 35-40 sujetos, aunque sólo se tuviese en cuenta en la evaluación final a los sujetos previamente seleccionados. Cada sesión duraba 50 minutos, el tiempo que duraba la clase. Se realizaron dentro del horario escolar, en horas de clase que correspondian a diversas asignaturas, cuyos profesores, bien porque se encontraban ausentes, bien por un acuerdo previo, habían cedido.

Como material de lectura para este entrenamiento se seleccionaron y adaptaron seis textos expositivos del propio material escolar del alumno y que pertenecían en su totalidad a la asignatura de Historia (adaptados del libro de texto de Prats y otros, 1987) ${ }^{1}$. En general, todos los textos poseian algunas características comunes, tales como la división del contenido en tres o cuatro párrafos o un nivel similar en su complejidad léxica y semántica; todos los textos de trabajo se presentaron con los mismos tipos de señalizaciones (frases previas a modo de resumen y señalizaciones numéricas). Todos ellos contenían, además, información relativa a la macroestructura y microestructura, y ninguno de ellos se presentaba con título. Los pasajes, sin embargo, diferían en su extensión que oscilaba entre 164 y 294 palabras, correspondiendo los más cortos a las primeras sesiones.

En estos seis pasajes se recogían cuatro de las cinco relaciones retóricas descritas por $\mathrm{Me}$ yer y cols. y que representaban la relación re- 
tórica de alto nivel en cada uno de ellos, a saber: Respuesta: Problema-solución y en otra forma, pregunta (textos 1 y 2, respectivamente), causación: antecedente-consecuente (textos 3 y 6), colección (texto 4) y descripción (texto 5). El hecho de utilizar dos textos para cada una de las relaciones de respuesta y causación y solamente uno para la colección y descripción, obedece al hecho de que se considera más importante estas dos primeras relaciones retóricas dominantes, como lo indica la estructura de contenido de una gran parte de la prosa expositiva. Por el contrario, la relación retórica de colección y descripción suele aparecer frecuentemente como elementos subordinados o aquéllas, y por consiguiente, no es usual encontrarlas como relaciones principales en este tipo de material escrito. El hecho intencionado por nuestra parte de no utilizar la relación retórica de comparación, frecuentemente utilizada en los trabajos de Meyer, obedece a razones que serán expuestas posteriormente.

\subsection{Diseño}

Se utilizó un diseño factorial mixto $2 \times 3 \times 2 \times 2$, con tres factores intergrupo (Grupo, Tratamiento y Ayuda del texto) y uno intrasujeto (Tarea de recuerdo). La mitad de los sujetos de cada nivel de competencia y tratamiento fue asignada aleatoriamente a cada una de las condiciones de señalización: versión señalizada (CS) y sin señalizar (SS).

\subsection{Evaluación}

La evaluación de los distintos tratamientos se realizó tras la lectura del texto «Orígenes del Descubrimiento de América». Su estructura organizativa fue elaborada de acuerdo al método de análisis propuesto por M=yer (1975, 1985b), sobre un texto original de Cespedes del Castillo $(1983)^{2}$. El pasaje contenía la relación retórica dominante de covarianza: antecedente-consecuente y contaba con dos versiones: Señalizada y no Señalizada. Tanto el procedimiento como la puntuación fueron idénticas al experimento anterior.

Para medir el recuerdo de cada lector, se siguió el análisis de la estructura del texto elaborada por Meyer $(1975,1985 b)$. Los datos finales de cada sujeto se agrupaban en torno a cuatro índices cuantitativos ( $A, B, R$ y $G$ ) y uno cualitativo $(O)$ (véase León $b$, en este mismo número).

\section{RESULTADOS}

\section{A. Puntuaciones A, B, R y G}

\subsection{Tratamientos}

Se observa una superioridad significativa en el recuerdo de la información relativa a las puntuaciones $\mathrm{R}$ de los lectores a los que se les instruyó mediante el tratamiento directo (ID) sobre los dos grupos control $(p<0.01)$. Como muestra la Figura 1, los lectores del grupo ID se mostraron más sensibles en la detección, retención y recuperación de las principales relaciones lógicas del texto (puntuaciones $R$ ) que sus compañeros de los grupos IC y SIC, lo que evidencia la eficacia de este programa de instrucción.

\subsection{Señalización}

Como se esperaba, los resultados revelan una superioridad en el recuerdo de los sujetos de la versión señalizada $(C S)$ sobre la no señalizada (SS) en las puntuaciones $A(p<0.05)$ y $R$ $(p<0.001)$. Esta superioridad de la versión $C S$ sobre la SS se produce prácticamente en todos los grupos y condiciones estudiados, aunque como veremos más adelante, depende de los factores intervinientes (nivel lector y tratamiento aplicado).

\subsection{Tarea de recuerdo libre: Inmediato/demorado}

Como estaba previsto, la cantidad de información en la tarea de recuerdo libre inmediata es claramente superior sobre la demorada en todas las puntuaciones y casos analizados $(p<0.001)$. Sin embargo, no se han encontrado diferencias significativas entre los distintos tratamientos aplicados y en ninguna de las tareas de recuerdo aplicadas.

\subsection{Análisis de las Interacciones}

Se han detectado un notable número de interacciones significaticas, especialmente en las puntuaciones $\mathrm{R}$, producidas por la confluencia de estos cuatro factores principales. Caben destacar las siguientes:

- Las producidas entre los factores Tratamiento-Señal-Recuerdo en $R(p<0.001)$ y $G(p<0.05)$. En este caso, los sujetos del tratamiento ID muestran un comportamiento diferente en el recuerdo de la información $\mathrm{R}$ ante la versión del texto y la tarea de recuerdo. Así y aunque la tendencia general sobre la cantidad de información recordada tiende a disminuir en la tarea de recuerdo como demora, esta ten- 


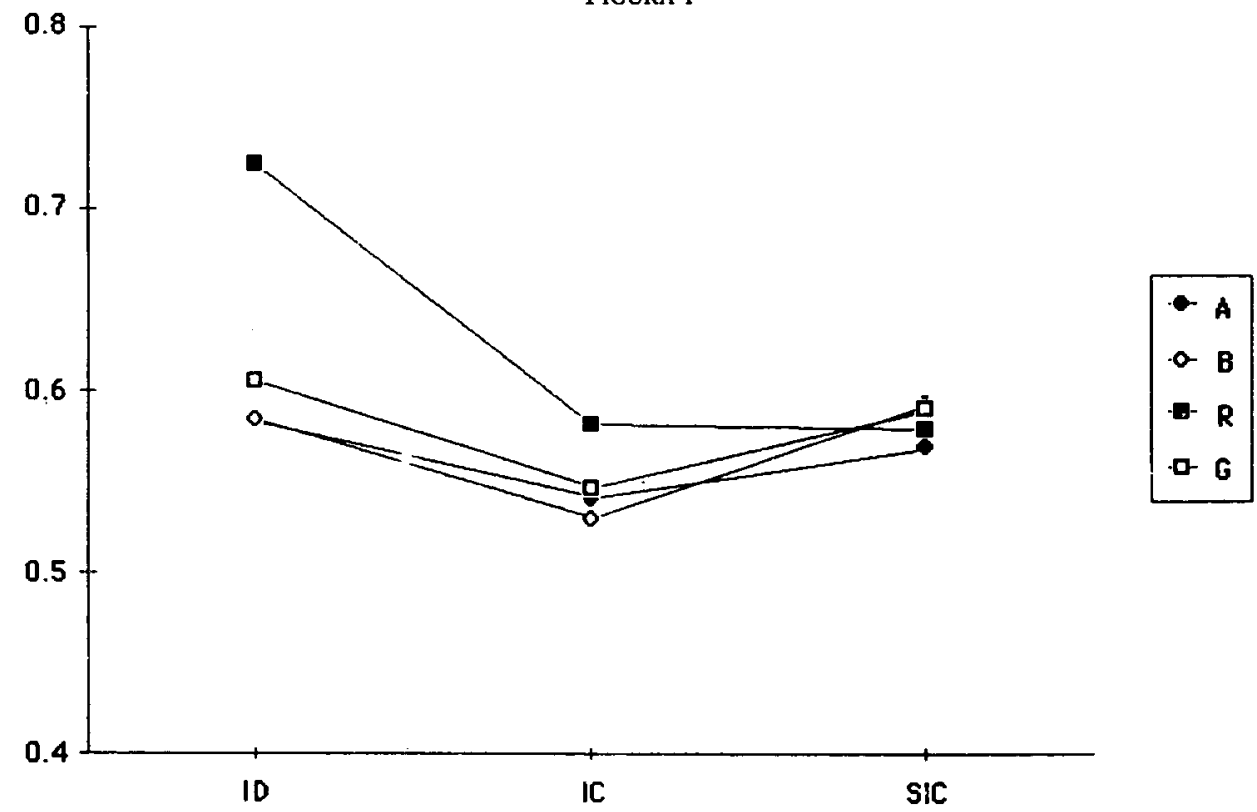

Puntuaciones medias obtenidas por los lectores de los diferentes tratamientos aplicados en cada una de las medidas realizadas $(A, B, R$ y $G) I D=$ Interv. directa. $I C=$ Interv. control. $S I C=G r u p o$ control sin intervención.

dencia se invierte en la versión no señalizada. En este caso, los lectores del tratamiento experimental parecen aplicar de manera diferente sus conocimientos y estrategias lectoras. Así y ante la ausencia de tales ayudas, los alumnos del tratamiento ID parecen llevar a cabo de un modo más estratégico su conocimiento sobre la estructura de los textos siendo capaces de retener la información cuando se les solicita una semana después. De hecho, esta habilidad de los sujetos del grupo ID para recuperar la información $\mathrm{R}$ en las situaciones más adversas (versión del texto no señalizada y tarea de recuerdo demorada), es realmente donde se producen las diferencias más importantes respecto a los grupos control, siendo ésta significativa en ambos casos $(p<0.05)$. Sin embargo, este comportamiento no se produce por igual en todos los componentes del tratamiento ID. Tal y como puede interpretarse de la interacción producida entre los factores Grupo-TratamientoSeñal en $R(p<0.01)$, son los alumnos BC de este tratamiento los que obtienen las mejores puntuaciones, siendo éstas significativas en el recuerdo de esta puntuación respecto a sus homólogos del grupo IC ( $\mathrm{p}<0.05)$.

\section{B. Puntuaciones 0: La organización del recuerdo}

Como puede observarse en la tabla 1.1 son los sujetos que han sido instruidos en el pro- grama de intervención directa los que mejor planifican sus respuestas siguiendo la estructura del texto en todas las situaciones estudiadas. En efecto, si tomamos las puntuaciones V y III conjuntamente, resulta que el grupo ID consigue el máximo porcentaje, siendo de un $75 \%$ $(29,2+45,8)$, seguido de los grupos control IC, con un $58,3 \%(12,5+45,8)$, y SIC, con un $54,1 \%(20,8+33,3)$.

Estas mejoras que obtienen los lectores del tratamiento ID se mantienen si consideramos a los sujetos BC y MC por separado. De esta manera, los sujetos $\mathrm{BC}$ del tratamiento experimental superan netamente a los lectores de ese mismo nivel de los grupos control, llegando a alcanzar un $91,6 \%(50+41,6)$ frente a un $74,9 \%(16,6+58,3)$ del grupo SIC y un $62,5 \%(29,2+33,3)$ del grupo IC. También los lectores MC del tratamiento experimental obtienen los mejores porcentajes, alcanzando un $58,3 \%(8,3+50)$ sobre los grupos control, $45,8 \%(12,5+33,3)$ para IC y $41,6 \%$ $(8,3+33,3)$ para el grupo SIC.

Otro dato de interés es el que se obtiene en las situaciones más adversas, en la versión no señalizada y en la tarea de recuerdo con demora. También en este caso son los lectores del tratamiento ID los que alcanzan los mejores porcentajes, situándose en un $83,2 \%$ $(41,6+41,6)$ para estos sujetos, en un $33,3 \%$ $(0+33,3)$ para la condición IC y en un $25 \%$ $(0+25)$ para los del grupo SIC. En este caso, 
TABLA I

Puntuaciones 0 obtenidas por los distintos grupos de lectores en el texto «Los orígenes del descubrimiento de Américas atendiendo a todos los factores analizados: grupo lector, tratamiento, señalización y tarea de recuerdo. Los datos se expresan en frecuencias y el valor que figura entre paréntesis expresa el porcentaje

\begin{tabular}{|c|c|c|c|c|c|c|c|c|}
\hline \multirow{3}{*}{ Grupo } & \multirow{3}{*}{ Punt. } & \multicolumn{2}{|c|}{ Rdo. inmediato } & \multicolumn{3}{|c|}{ Rdo. demorado } & \multirow{3}{*}{ Total BC } & \multirow{3}{*}{$\begin{array}{c}\text { Total MC Total GLO- } \\
\text { BAL }\end{array}$} \\
\hline & & Sujetos BC & Sujetos MC & Suje & $B C$ & Sujetos MC & & \\
\hline & & CS SS & SS & CS & SS & CS & & \\
\hline
\end{tabular}

ID

$\begin{array}{rrrrrrrrrrrrr}\text { V } & 6 & 1 & 1 & 0 & 0 & 5 & 1 & 0 & 12(50.0) & 2 & (8.3) & 14(29.2) \\ \text { III } & 0 & 4 & 3 & 1 & 5 & 1 & 4 & 4 & 10(41.6) & 12(50.0) & 22(45.8) \\ \text { I } & 0 & 1 & 2 & 5 & 1 & 0 & 1 & 2 & 2 & (8.3) & 10(41.6) & 12(25.0)\end{array}$

IC

$\begin{array}{rlllllllllll}\text { V } & 5 & 0 & 2 & 0 & 2 & 0 & 1 & 0 & 7(29.2) & 3(12.5) & 10(20.8) \\ \text { III } & 1 & 4 & 3 & 3 & 0 & 3 & 1 & 1 & 8(33.3) & 8(33.3) & 16(33.3) \\ \text { I } & 0 & 2 & 1 & 3 & 4 & 3 & 4 & 5 & 9(37.5) & 13(54.2) & 22(45.8)\end{array}$

SIC

\begin{tabular}{rrrrrrrrrrrrr} 
V & 2 & 0 & 1 & 0 & 2 & 0 & 1 & 0 & $4(16.6)$ & $2(8.3)$ & $6(12.5)$ \\
III & 3 & 5 & 4 & 0 & 3 & 3 & 4 & 0 & $14(58.3)$ & $8(33.3)$ & $22(45.8)$ \\
I & 1 & 1 & 1 & 6 & 1 & 3 & 1 & 6 & $6(25.0)$ & $14(58.3)$ & $20(41.7)$ \\
\hline
\end{tabular}

ID = Intervención directa; IC = Intervención control; SIC = Grupo control sin intervención; CS = Versión señalizada; SS $=$ Versión sin señalización.

las diferencias entre los tratamientos aumentan de manera importante. Obsérvese que más del $40 \%$ de los sujetos de la condición ID han sido capaces de organizar su recuerdo siguiendo de manera explícita la estructura original del texto (puntuación V) frente a un $0 \%$ de los sujetos de los grupos control.

\section{DISCUSION}

Los resultados obtenidos en este estudio se acomodan no sólo a la caracterización que habíamos establecido previamente entre los distintos niveles de competencia lectora, sino en la posible modificación de sus estrategias lectoras a través de este modelo de intervención. Así, los sujetos que recibieron el tratamiento ID incrementaron significativamente su recuerdo en las puntuaciones $\mathrm{R}$ sobre los dos grupos control ( $p<0.01)$. Esta mejora selectiva en las relaciones lógicas que gobiernan la estructura de los textos expositivos, es en el único caso que se mantiene con independencia a la versión del pasaje aplicado o a la tarea de recuerdo asignada. Este hecho, sin embargo, recae fundamentalmente en los $\mathrm{BC}$, ya que en los sujetos $\mathrm{MC}$, aunque obtienen algunas mejoras respecto a sus compañeros de los grupos control, éstas no llegan a ser en todos los casos significativas.
Respecto a las señalizaciones, tanto los sujetos BC y MC de este tratamiento mejoran su recuerdo de la información relativa a estas mismas puntuaciones R. Los lectores de este tratamiento obtienen también los mejores resultados en las puntuaciones $R$ en la tarea de recuerdo inmediata sobre los sujetos del grupo SIC, y es prácticamente en todas las medidas realizadas en la tarea con demora, siendo significativas sobre el tratamiento IC $(\mathrm{p}<0.001)$. Cuando a estos lectores se les presenta la versión no señalizada, acusan en un primer momento la ausencia de tales ayudas, pero mejoran considerablemente en la segunda tarea de recuerdo. Esta es una característica que sólo se observa en los sujetos BC de este tratamiento.

Sobre la organización del recuerdo, también son los lectores del tratamiento ID, tanto los $\mathrm{BC}$ como los $\mathrm{MC}$, los que más frecuentemente utilizan el plan organizacional del texto en la elaboración de sus respuestas. Estas diferencias aumentan en la versión no señalizada y en el recuerdo con demora.

Todos estos resultados, muy vinculados entre sí, como lo han demostrado el importante número de interacciones que se han producido entre las variables aplicadas, indican que los sujetos que intervinieron en el programa de intervención experimental, especialmente los más capaces (BC), han utilizado la estrategia estruc- 
tural para identificar y aplicar las relaciones dominantes del texto en la elaboración de sus respuestas cuando tratan de recordar la información de un texto expositivo, aunque, como es el caso, requiera un mayor conocimiento específico sobre la estructura de los textos que ellos no poseían hasta este entrenamiento. Este programa de intervención, por tanto, proporciona a los sujetos, especialmente a los que ya poseen una habilidad comprensiva aceptable (BC), una mejora considerable en la calidad del producto final lector, de manera similar a como lo realizaron los lectores más expertos en las puntuaciones $R$ sobre este mismo texto (véase el estudio anterior, en esta misma revista). Este comportamiento se caracteriza por una mayor habilidad en la detección de la información relevante, mayor estabilidad del recuerdo con el paso del tiempo y en la frecuencia con la que siguen la estructura del pasaje cuando los lectores elaboran sus respuestas.

A pesar de todo, este modelo de intervención no ha cumplido todos los objetivos propuestos. El modelo de instrucción ID no da cuenta de la mejora en los sujetos MC. En este caso, aunque se observan algunas mejorías respecto a sus compañeros de los grupos control, la intervención no ha resultado del todo satisfactoria.

\section{Estudio 2}

\section{PLANTEAMIENTO DE LA INVESTIGACION}

Este estudio es en realidad, una prolongación del anterior. En él nos cuestionábamos la generalidad del programa de instrucción ID. Nos preguntábamos cómo los diferentes grupos de sujetos que participaron en el estudio anterior se comportarían ante una situación nueva, ante la presentación de un texto organizado por una relación retórica (Comparación) no conocida, no explicada en ningún momento anterior. Esta fue la razón por la que en el programa de intervención ID no se incluyó esta relación retórica. Por otro lado, algunos trabajos precedentes a éste han resaltado una diferencia importante entre el recuerdo de la información del texto y el aprendizaje del mismo (Kinstch, 1986; Mannes y Kintsch, 1987). En este sentido, un entrenamiento previo en estrategias lectoras y orientado hacia lo que va a ser estudiado inmediatamente después, puede ayudar a los lectores a comprender y recordar mejor el material escrito. Sin embargo, si ese entrenamiento se organiza de manera diferente a la tarea que se le va a solicitar después, los re- sultados que se obtienen pueden ser mejores cuando se le exije al sujeto aplicar al lector lo aprendido ante situaciones nuevas.

Nuestro propósito en esta investigación, por tanto, no fue otro que el tratar de comprobar experimentalmente si los lectores del tratamiento ID serían capaces de aplicar sus nuevos conocimientos sobre la estructura del texto en la detección, asimilación y recuperación de la información leída de un texto que no corresponde exactamente a lo aprendido. En tal caso, se detectarían diferencias significativas entre este grupo de intervención directa y los dos grupos control.

\section{METODO}

\subsection{Sujetos y tratamientos}

Los sujetos que participaron en este estudio fueron los mismos del estudio anterior, un total de 72 , distribuidos en las mismas condiciones experimentales.

\subsection{Materiales}

Para medir la capacidad de generalización de los tratamientos, se utilizó un tercer texto, "LOS INICIOS DEL FERROCARRIL EN AMERICA", correspondiente a una versión española de la utilizada por Meyer $(1984,1985 a$ y b). Sus características son similares a las de los ya utilizados: tiene una extensión de 434 palabras, dispone de 10 niveles y su nivel de dificultad comprensiva es similar a la del texto "Superpetroleros" utilizado en otros estudios. La diferencia respecto a los demás textos estriba en su relación retórica dominante, una Comparación Adversativa, en torno a la cual se organiza todo el contenido del texto. Para este estudio sólo se utilizó la versión señalizada.

\subsection{Diseño y Evaluación}

Para analizar los datos relativos a este nuevo pasaje se elaboró un diseño factorial $2 \times 3$ con dos factores intersujetos (lector y tratamiento). Se computaron un total de cuatro ANOVAs, uno para cada una de las variables medidas: A, $B, R$ y $G$. Como en los estudios anteriores, se dispuso de otro apartado en el que se consideraron las puntuaciones cualitativas 0 . En este estudio sólo se consideró la tarea de recuerdo libre inmediato. $\mathrm{La}$ evaluación del recuerdo, procedimiento y puntuación utilizados fueron idénticos a los análisis ya realizados en el estudio anterior. 


\section{TABLA II}

Contraste de medias correspondiente a los distintos tratamientos aplicados ID, IC y SIC, tomados globalmente en cada una de las puntuaciones analizadas. El nivel de significación $\mathrm{p}$ ha sido hallado según Scheffé. Los espacios señalados con guiön indican una ausencia de significación

\begin{tabular}{lcccc}
\hline & A & B & R & G \\
\hline ID > IC & 0.001 & - & 0.01 & 0.001 \\
ID > SIC & 0.0001 & - & - & - \\
SIC > IC & - & 0.05 & - & - \\
\hline
\end{tabular}

\section{TABLA III}

Puntuaciones 0 obtenidas por los distintos grupos de lectores en el texto *Los inicios del ferrocarril en América * atendiendo a los dos factores analizados: grupo lector y tratamiento.

Los datos se expresan en frecuencias y el valor que figura entre paréntesis expresa el porcentaje

\begin{tabular}{lrrrr}
\hline \multicolumn{1}{c}{ Programa } & Punt. & Sujetos BC & Sujetos MC & Total GLOBAL \\
\hline ID & & & & \\
& V & $9(75.0)$ & $2(16.6)$ & $11(45.8)$ \\
& III & $3(25.0)$ & $7(58.3)$ & $10(41.6)$ \\
& I & $0(0.0)$ & $3(25.0)$ & $3(12.5)$ \\
IC & & & & \\
& V & $3(25.0)$ & $0(0.0)$ & $3(12.5)$ \\
& III & $6(50.0)$ & $7(58.3)$ & $13(54.1)$ \\
SIC & I & $3(25.0)$ & $5(41.6)$ & $8(33.3)$ \\
& & & & \\
& V & $6(50.0)$ & $1(8.3)$ & $7(29.1)$ \\
& III & $5(41.6)$ & $4(33.3)$ & $9(37.5)$ \\
& I & $1(8.3)$ & $7(58.3)$ & $8(33.3)$ \\
\hline
\end{tabular}

ID = Intervención directa; IC = Intervención control; SIC = Grupo control sin intervención.

\section{ANALISIS DE LOS RESULTADOS}

A) Puntuaciones A, B, R y G

\section{Tratamiento}

Como puede observarse en la tabla II, las puntuaciones obtenidas por el grupo que recibió intervención directa (ID) fueron netamente superiores al resto de los grupos en las puntuaciones $A(p<0.001)$, y superiores, aunque sólo significativas sobre el grupo de Intervención Control (IC), en las puntuaciones $R$ ( $\mathrm{p}<0.01$ ).

$\mathrm{Si}$ analizamos los datos de una manera más pormenorizada, esta vez en función no sólo del tratamiento aplicado sino también del nivel lector, se detecta un incremento importante en el recuerdo de la información esencial del texto tanto de los sujetos BC como de los MC que recibieron el tratamiento ID sobre sus respectivos compañeros de los grupos control. En efecto, existen diferencias significativas entre el recuerdo de los lectores $B C$ de tratamiento experimental y los lectores de este mismo nivel lector de los grupos control en las puntuaciones A $(p<0.001)$. De la misma manera, el recuerdo de los sujetos MC del tratamiento ID se muestra superior al de los grupos control en la información relativa a esta misma puntuación, aunque esta vez sólo significativa sobre el grupo IC (A: $p<0.05)$.

\section{B) Puntuaciones cualitativas 0}

Tal y como se expresa en la tabla III, también se corrobora esta superioridad del tratamiento experimental sobre los grupos control en la utilización del plano organizacional en la elaboración del recuerdo. En este sentido, las puntuaciones globales indican que los lectores del tratamiento ID siguen con mayor frecuencia la estructura del texto en la elaboración de 
su recuerdo escrito. Tomando conjuntamente las puntuaciones V y III, los sujetos del tratamiento experimental obtienen los porcentajes más altos con un $97,4 \%(45,8+41,6)$, seguido del grupo SIC, con un $66,6 \%(29,1+37,5)$ y del tratamiento IC, también con un $66,6 \%$ $(12,5+54,1)$.

Esta superioridad en la utilización del plan del texto en la elaboración del recuerdo también queda reflejada si añadimos, además, el nivel lector. Así, en el caso de los lectores BC, son los que pertenecen al tratamiento ID los que obtienen los mejores resultados, esta vez de un $100 \%(75+25)$, frente a un $91,6 \%$ $(50+41,6)$ del grupo SIC y un $75 \%(25+50)$ de los sujetos del tratamiento IC. Resulta interesante resaltar que en este caso, a pesar de que los porcentajes son más parejos entre los distintos grupos, las $3 / 4$ partes de las respuestas tabuladas de los sujetos del tratamiento experimental han seguido explicitamente la estructura principal del texto enunciando además sus elementos principales.

De la misma manera, los sujetos MC del grupo ID son los que mejor han organizado su recuerdo, un $74,9 \%(\mathbf{1 6 , 6}+\mathbf{5 8 , 3})$, frente a un $58,3 \%(0+58,3)$, de los sujetos del grupo IC y un $41,6 \%(8,3+33,3)$, de los del grupo SIC.

\section{DISCUSION GENERAL}

Los resultados obtenidos reflejan que los lectores que fueron entrenados mediante el modelo de Instrucción ID, han sabido aplicar sus conocimientos sobre la estructura del texto a otro cuya relación retórica dominante y sus principales elementos no habían sido previamente instruida. Esta transferencia de lo aprendido a situaciones nuevas se manifiesta en:

- El incremento significativo del recuerdo de las ideas esenciales del texto y en los dos niveles de competencia sobre los grupos control.

- En un decremento de la información de detalle (B).

- En la mayor utilización por parte de estos lectores del plan organizativo del texto en la elaboración del recuerdo.

Un efecto llamativo en esta transferencia ha sido la menor significación que el grupo ID ha obtenido sobre los dos control en el recuerdo de las relaciones lógicas dominantes de la estructura. Este hecho producido quizás por ese desconocimiento previo sobre esta organización del pasaje, ha producido, sin embargo, un incremento significativo de la información relevante del texto, de la macroestructura (A).

Con este último dato deseamos poner de relieve lo :que podría considerarse como una li- mitación más profunda de este trabajo: que la comprensión no es una mera copia de la semántica del texto en la memoria, a pesar de que hemos actuado como si lo fuera. Podría considerarse que el tipo de representación que aquí inducimos es del tipo «semántico-proposicional» y que con ello no se asegure una adecuada representación «situacional» en términos de Perrig y Kintsch (1985). El hecho de que el lector modifique o transfiera los conocimientos intruidos de manera relativamente diferente a como se les intruyera previamente ante situaciones nuevas, nos alienta a considerar que ello suponga un paso para poder reconstruir una representación sobre el mundo o sobre la situación que se evoca en el texto (van Dijk y Kintsch, 1983).

\section{CONCLUSIONES}

En definitiva, los resultados de estos dos experimentos nos permiten defender las siguientes conclusiones:

1. En general, los sujetos que se sometieron al programa de instrucción ID obtuvieron mejoras significativas en el recuerdo respecto a los grupos de control.

2. Estas mejoras han sido selectivas en el sentido de que sólo afectan a aquellos aspectos que guardan relación con las estrategias instruidas. Más específicamente, estas ganancias se han obtenido en:

- El recuerdo de las relaciones lógicas que gobiernan la estructura de los textos expositivos con independencia a la versión del pasaje aplicado o a la tarea de recuerdo.

- El recuerdo de las ideas centrales (y no de detalle) del texto.

- La mayor estabilidad del recuerdo de la información relevante ante la tarea de recuerdo demorada.

- Una mayor sensibilidad hacia las señalizaciones del texto.

- El seguimiento del plan organizacional del texto en la elaboración de sus respuestas. Se detecta una organización del recuerdo basa$\mathrm{da}$ en la propia lógica del texto.

3. Estas habilidades se generalizan a textos cuya estructura organizativa no había sido desvelada $o$ instruida con anterioridad.

Todos estos cambios que observamos en los sujetos instruidos con este modelo de intervención corresponde a lo que podríamos deducir si se abandonara la estrategia de listado por la estrategia estructural: aplicar sus conocimientos sobre la organización interna del texto tanto en la detección, asimilación, recuperación y organización de las respuestas sobre la infor- 
mación leída, como en la transferencia de esos conocimientos a situaciones nuevas. De esta forma, este nuevo comportamiento lector se aproxima al utilizado por los más expertos.

Este modelo de instrucción conlleva, sin embargo, algunas limitaciones. El modelo, aunque mejora significativamente el rendimiento lector en los alumnos competentes, no mejora de manera significativa en todos los casos, el rendimiento de los lectores menos capaces. Existen al menos dos aspectos que pueden haber influido en la eficacia del programa. El primero, hace mención al número de sesiones administradas (cinco). Puede que este número de sesiones haya resultado insuficiente modificar adecuadamente sus estrategias lectoras y aplicarlas ante la lectura de la nueva información. Un segundo factor hace referencia al número de sujetos que participaron en la intervención. Puede que en este caso el número resultase exce- sivo como para asegurar que la aplicación del programa ha sido llevada adecuadamente y por igual en todos los sujetos. En todo caso, estos problemas quedan abiertos a posibles investigaciones futuras que permitan esclarecer de una manera más precisa las causas de este hecho.

\section{Notas}

${ }^{1}$ Los textos utilizados en los programas de insvizcción pertenecen al libro de texto, Prats, J., Castelló, J. E., García, M. C., Izuzquita, I y Loste, M. A. (1987). Historia. Bachillerato 1. Madrid: Anaya.

2 Este texto puede localizarse en Céspedes del Castillo, G. (1983). América hispánica (1492-1898). En M. Tuñón de Lara (Ed.). Historia de España, tomo VI. Barcelona: Labor.

\section{Referencias}

BARTLETT, B. J. (1978). Top-level structure as an organizational strategy for recall of classroom text. Tesis doctoral no publicada. Arizona State University.

BROOKS, L. W. y DANSEREAU, D. F. (1983). aEffects of structural schema training and text Organization on Expository Prose Processing". Journal of Educational Psychology, 75, 6, 811-820.

Brown, A. L., DAY, J. D. y JONES, R. S. (1983). *The Development of Plans Summarizing Texts». Child Development, 54, 968-979.

Brown, A. L., PALINCSAR, S. A. (1982). «Inducing strategic learning from texts by means of informed, selfcontrol training. Topics in Learning and Leaming Disabilities, 2, 1-17.

CARRETERO, M. y LEÓN, J. A. (1990): «Desarrollo cognitivo y aprendizaje en la adolescencia». En J. Palacios (Comp), Psicologia Evolutiva. Madrid: Alianza.

GEVA, E. (1983). «Facilitating reading through flowcharting». Reading Research Quarterly, 18, 384-405.

VAN DijK, T. A. y KINTSCH, W. (1983). Strategies of discourse comprebension. New York: Academic Press.

JUST, M. A. y CARPENTER, P. A. (1987). The Psychology of Reading and Language Comprehension. Massachusetts: Allyn and Bacon.

KINTSCH, W. (1986). «Learning from textn. Cognition and Instruction, 3, 87-108.

LEON, J. A. (1989): Memoria y comprensión de textos: Diferencias entre sujetos expertos y novatos. Tesis doctoral inédita. Universidad Autónoma de Madrid.

LEÓN, J. A. Y CARRETERO, M. (1990): *Intervention in comprebension strategies: Knowledge and use of the text structure. Paper presentado en European Association for Research on Learning and Instruction. Workshop aText Proccesing from different perspectives. Universidad de Amsterdan. Holanda.

LeÓN, J. A. Y Garcia MAdRugA, J. A. (1989): “Comprensión de Textos e Instrucción». Cuadernos de Pedagogia, 169, 54-59.

LOMAN, N. C. Y MAYER, R. (1983). «Signaling Techniques that increase the understability of expository prose». Joumal of Educational Psychology, 7S, 402-412.

MANNES, S. M y KINTSCH, W. (1987). «Knowledge Organization and Text Organization». Cognition and Instruction, 4 (2), 91-115.

MAYER, R. E. (1985). «Structural analysis of Science prose: can we increase problem-solving perfomance». En B. K. BRITTON y J. B. BLACK: Understanding expository text. Hillsdale, Nueva Jersey: Erlbaum.

MEYER, B. J. F. (1975). The organization of prose and its effects on memory. Amsterdam: North-Holland.

- (1984). «Text dimensions and cognitive procesing*. En H. Mandl, N. L. Stein, \& T. Trabasso (Eds.), Learning and comprebension of text. Hillsdale, NJ: Erlbaum.

- (1985a). «Prose analysis: Purposes, procedures and problems». En B. K. Britton \& J. B. Blanck (Eds.), Understanding Expository Text. Hillsdale, Nueva Jersey: Erlbaum.

- (1985b). «Prose analysis: Purposes, procedures and problems (Part. IIn). En B. K. Britton \& J. B. Black (Eds.), Understanding Expository Text. Hillsdale, Nueva Jersey: Erlbaum.

MEYER, B. J. F., YOUNG, C. J. y BARTLETT, B. J. (1989). Memory Improved. Reading and Memory Enhancement Across the Life Span Through Strategic Text Structures. New Jersey: Erlbaum.

OAKHILl, J. y GARNHAM, A. (1988). Becoming a Skilled Reader. Nueva York: Basil Blackwell. 
PALINCSAR, A. S. y BROWN, A. L. (1984). *Reciprocal teaching of comprehension-fostering and comprehension-monitoring activitesw. Cognition and instruction, 1, 117-175.

PERRIG, W. y KINTSCH, W. (1985). *Propositional and situational representation of text*. Journal of Memory and Language, 24, 503-518.

SCARDAMALIA, M. y Beremter, C. (1984). «Development of strategies in text processing». En H. Mandl, N. L. Stein y T. Trabasso. Leaming and comprebension of text. Hillsdate. Nueva Jersey: Erlbaum.

SPRING, C. (1985). «Comprehension and study strategies reported by university freshmen who are good and poor readers". Instructional Science, 14, 157-167.

TAYLOR, B. M. (1980). "Children's memory for expository text after reading». Reading Research Quarterly, 15, 399-411.

TAYLOR, B. M. y BEACH, R. W. (1984). *The effects os text structure instruction on middle grade students comprehension and production of expository text". Reading Research Quarterly, 19, 134-146.

WILIIAMS, J. P., TAYLOR, M. B. y GANGER, S. (1981). "Text variations at the level of the individual sentence and the comprehension of simple Expository Paragraphs». Journal of Educational Psychology, 73, 6, 851-865.

\section{Extended Summary}

Recent studies suggest that the good readers seem to be more active than those readers with a poor level of comprehension when they process the information of the text. So, from this perspective, the actual strategies of the competent readers have seen identified and named in different forms: *organizational strategy» (Spring, 1985), «meaningful strategy (Loman \& Mayer, 1983), assimilative strategy (Mayer, 1985) or *structural strategy* (Meyer, 1984). These strategies are intented to activate the schemes that the subject possesses. Also they require of the reader the existence of previous adequate knowledge and the possibility of the strategid use of that knowledge.

On the other hand, the poor reader presents others types of strategies, like the "rote reading strategy» (Loman \& Mayer, 1983), «default strategy» (Meyer, 1984) or when confronted with summary tasks, the «copy-delete strategy» (Brow, Day \& Jones, 1983). These strategies are characterized by a fragmented and linear representation and are utilized when the reader conceives of the passage as a list of separate actions and events that must be memorized.

These differences suggest to us, at the time of planning an instructional and instructional model that influences the improvement of the comprehension of written material by less competent readers, that we should base our assumption on the fact these readers can be benefit from the teaching of those strategies that tme mature readers used spontaneously. So, in tw complimentary studies, the implantation and later the evaluation of an instructional program of high school students with different reading levels was analyzed. This program called Direct Intervention (DI) basically influenced the strategies that were required for its application of an explicit knowledge about the structure of the text, particularly that relative to the principle logical experimental program was also supported in a procedure that combine the explanation, the modelling and the practice directed fases in such strategies.

In the first study, the 72 junior high school students (ages 14-15 years old), that participated and who differed in level comprehension, 36 with a good level (GC) and 36 subjects with a poor reading comprehension level (PC) were evenly distributed into three gorups. The first group was the experimental group who participated in the mentioned program and the other two control groups. In order to evaluate the performance of the groups, two tests of free recall, immediate and delayed (seven days), were given. The results obtained reflect a substantial improvement of the readers who were trained in this program in respect to their homologous partnes in the control group in: 
- The recall of the logical relationships that govern the structure of the text.

- The recall of the central ideas (and not of detail) of the text.

- The greatest amount of stability of recall of the relevant information when confronted with a delayed recall task.

- The following of the organizational plan of the text in the elaboration of their reponses.

- In the second study, the trained subjects in experimental progra transfered their new knowledge to text whose organizational structure hat not been revealed or instructed earlier.

\section{Anexo}

Recuadro gráfico utilizado en tratamiento directo (ID) en los textos cuya relación dominante se identificaba con la respuesta o la causación

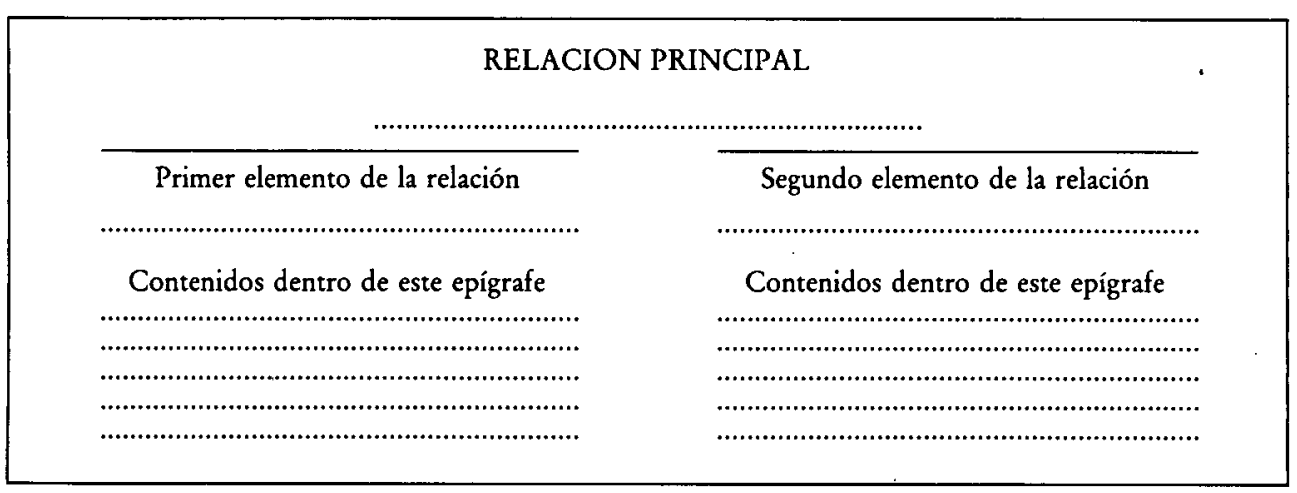

